\title{
Consideraciones teóricas y éticas del reconocimiento facial de las emociones en contexto de pandemia
}

\author{
CRISTHIAN ALMONACID DÍAZ* \\ Universidad Católica del Maule (Chile) \\ calmonacid@ucm.cl
}

\begin{abstract}
Resumen
El reconocimiento facial es una tecnología que se ha utilizado para el control de la población a fin de combatir los efectos de la pandemia por COVID-19. Sin embargo, esta tecnología se utiliza hace bastante tiempo en muchos ámbitos del quehacer económico, social y político. Este trabajo analiza el reconocimiento facial entendida como la captura de micro expresiones faciales mediante la cual se puede medir y evaluar emociones con el fin de vigilar movimiento, predecir comportamientos y anticipar intenciones de compra y de voto. Nuestro objetivo es revisar los enfoques teóricos que sustentan esta tecnología que ha devenido en una nueva oportunidad de negocio, para proponer después algunas reflexiones éticas derivadas.
\end{abstract}

Palabras claves: Reconocimiento facial, negocio emocional, emociones, neuromarketing, neuroeconomía.

\section{Theoretical and ethical considerations of facial recognition of emotions in the context of a pandemic}

\begin{abstract}
Facial recognition is a technology that is used to control people in order to fight the impacts of COVID-19. However, this technology has been used for many years in many economical, social and political areas. This work provides an analysis of the new business based on technologies of facial micro-expressions recognition. By means of this procedure, the involved companies measure and evaluate emotions with the aim of monitoring the movement, predicting behaviors, intentions of purchase and vote, between other applications. Our purpose is to check the theoretical suppositions that sustain this business, to propose later some ethical derivative reflections.
\end{abstract}

Key words: Facial recognition Emotional business, emotions, neuromarketing, neuroeconomics.

\footnotetext{
* Dr. en Filosofía, especialidad en Ética y Democracia por la Universidad de Valencia, España, Máster en Ética y Democracia por la Universidad de Valencia y Magíster en Filosofía por la Pontificia Universidad Católica de Valparaíso, Chile. Es profesor auxiliar en el Departamento de Filosofía de la Facultad de Ciencias Religiosas y Filosóficas de la Universidad Católica del Maule, Talca, Chile. Sus principales líneas de investigación son: ética, éticas aplicadas, emociones y racionalidad práctica, ciudadanía y democracia.
} 
En diferentes medios de comunicación se ha destacado la capacidad de control digital de la población en los países asiáticos para mantener a raya los efectos de la pandemia. Efectivamente, en países como China, Corea o Japón la implementación de la tecnología de reconocimiento facial para el seguimiento de los contagiados de COVID-19 fue una de las medidas más exitosas. Dicho procedimiento vino a demostrar que la vigilancia tecnológica puede servir para salvar vidas. Entre los factores de este éxito se encuentra cierta cultura y disposición de la población en aquellas latitudes para estar permanentemente sometida a observación y control de sus libertades en pos de la organización de lo común. Las cámaras de vigilancia son habituales y están repartidas por millones. Muchas de estas cámaras están provistas con las más actuales técnicas de reconocimiento facial, pensadas para inspeccionar detalles físicos, examinar temperatura corporal, identificar ocularmente a los individuos, etc. La experiencia en los países asiáticos es que nada hay que pueda escaparse al control de una población dispuesta incluso a ser vigilada desde su teléfono móvil.

Lo cierto es que esta tecnología no es algo nuevo. Hace bastante tiempo, en diferentes lugares del mundo han emergido emprendimientos empresariales que han desarrollado todo tipo de aplicaciones en base a biométricas que permiten indagar diferentes aspectos de la vida y movimientos de las personas a partir de las manifestaciones físicas de las emociones. Estos procedimientos se han incorporado a las estrategias de seguridad pública e inteligencia estatal, se disponen en centros comerciales y se utilizan en diferentes países para la observación de la actividad social y política de los ciudadanos. De manera que la pandemia ha resultado ser un aliciente más en el fortalecimiento y extensión a otros campos de aplicación de esta tecnología, que está resultando altamente lucrativa para empresas especializadas en el tema.

En este trabajo pretendemos revisar las bases teóricas que determinan el procedimiento del reconocimiento facial gracias a las expresiones musculares que provocan las emociones, para reflexionar sobre las implicancias éticas de esta tecnología en la medida que plantea una serie de cuestiones relativas a la privacidad, la manipulación o la toma de decisiones sin consentimiento de los afectados. Para alcanzar este objetivo, en un primer momento describimos en qué consiste esta tecnología para de la medición de emociones vía reconocimiento facial. Después analizamos los componentes teóricos de la perspectiva fisiologista de las emociones. En un segundo momento confrontamos la perspectiva fisiologista con una pers- 
pectiva cognitiva fisiológica, más atenta a la intencionalidad y la interpretación que acompaña a las emociones. Finalmente, derivamos algunas reflexiones éticas y nuestras conclusiones al respecto.

\section{LAS NUEVAS OPORTUNIDADES DE NEGOCIO A PARTIR DE LOS AVANCES DEL NEUROMARKETING: EL CASO DE LAS EMPRESAS ESPECIALIZADAS EN MEDIR EMOCIONES MEDIANTE RECONOCIMIENTO FACIAL}

La más novedosa y controvertida contribución de la nueva ciencia de la neuroeconomía afirma que el consumidor no compra mediante la activación cognitiva prefrontal, sino que cualquier acción de compra viene determinada por la parte límbica del cerebro. En otras palabras, los estudios en neuroeconomía vendrían a demostrar que los consumidores compran teniendo en cuenta principalmente factores emocionales, por ende, se parte del supuesto que el consumidor toma decisiones irracionales (Cisneros, 2015).

Siguiendo este horizonte, asociaciones globales, como por ejemplo Neuromarketing Science \& Business Association (NMSBA) ${ }^{1}$, han fusionado a las neurociencias, la economía y el marketing, para convocar a investigadores interesados en analizar el comportamiento del consumidor desde la óptica del neuromarketing (Lee, Broderick \& Charberlain, 2007). El objetivo de estos centros de investigación es estudiar, desde un punto de vista científico neuronal, la dimensión emocional del cerebro en la toma de decisiones del consumidor. Como resultado se ha comprobado que el cerebro, en su funcionamiento habitual, toma diferentes atajos para acelerar el proceso de decisión de compra. Esto quiere decir que el cerebro no siempre se detiene a operacionalizar decisiones mediante elaboraciones estrictamente racionales (Conill, 2012: 51; Gigerenzer, 2008). Además, y lo más interesante para nuestro trabajo, se ha afirmado que estas decisiones de compra no se dan en una caja negra inaccesible, pues dichas decisiones sin elaboración racional se pueden observar y analizar mediante diferentes mecanismos de imagen neurocerebral u observación facial y ocular (Lindstrom, 2010; Racine, Bar-ilan \& Illes, 2005; Dimigen, Sommer, Hohlfeld \& Klieg, 2011). Estas mediciones permiten establecer biométricas de fácil aplicación en pos de hacer más eficiente la comercialización de un producto o para calcular intenciones de voto (Cortina, 2011: 99-102).

El neuromarketing, bajo estos presupuestos, se ha erigido un campo decisivo para el éxito comercial, siendo capaz de desarrollar estrategias y herramientas publicitarias apoyadas en diferentes mecanismos, con base científica y neurobiológica. Su objeto de conocimiento es influir en los

Se puede consultar en el siguiente enlace disponible en: http://www.nmsba.com/ 
consumidores, aumentar la efectividad comercial (MacInnes \& Steidl, 2016) y mejorar los beneficios de aquellas empresas que buscan posicionar sus marcas y sus productos en el mercado (Klarick, 2016).

Dentro de este panorama y en consideración a cómo los consumidores actúan guiados emocionalmente a la hora de comprar, han emergido diferentes startups dedicadas a crear softwares especializados en el reconocimiento de emociones mediante la "lectura" de microexpresiones faciales que se obtienen por webcam o videoconferencias. A partir de esa información desarrollan algoritmos que les permite acceder a los estados de ánimo de diferentes individuos mientras ven anuncios, debates políticos o programas de TV. Con los resultados predicen el nivel de aceptación, visualizan las reales posibilidades de compartir determinados contenidos o medir las intenciones de compra o voto. Este desarrollo tecnológico que dice ser fehaciente y asertivo, permite aplicaciones concretas en los campos de la investigación de mercado comercial o laboral, en el marketing, en la política y recientemente en el control digital de la población para disminuir los efectos de la pandemia.

Entre estas nuevas empresas podemos mencionar a: Emotion Research Lab (García, 2016: 51), Eyeris y Affectiva ${ }^{2}$. Estas empresas también proveen su tecnología a instituciones gubernamentales, a diferentes empresas, para complementar procesos de seguridad pública o para la selección de personal a través de análisis de videos-currículum que permiten reconocer el nivel de confiabilidad de los postulantes a un puesto de trabajo. También se conocen aplicaciones en los emprendimientos de producción audiovisual, en la medida que el reconocimiento facial aseguraría poder discriminar a partir de la observación de los evaluados el tipo de imagen que causa mayor impacto emocional y, por ende, aquellas imágenes que serán más exitosas, dependiendo de lo que se quiera vender.

La base del negocio, como podemos observar, es la capacidad de desarrollar una suerte de "máquina de la verdad" que descubre lo que siente un sujeto a partir de sus microexpresiones faciales para poder conocer su susceptibilidad a determinada decisión o comportamiento. A este conocimiento se puede acceder incluso antes de lo que el sujeto puede manifestar voluntariamente. A partir de esos datos se definen estrategias de comunicación en campañas de marketing o en campañas electorales, pues los datos obtenidos entregan un paneo de los modos en que se comportan consumidores y votantes, midiendo sus emociones en tiempo real a fin de

2 Se puede consultar directamente la página web de la empresa Emotion Research Lab, disponible en: http:/ /www.emotionresearchlab.com/ Otras empresas dedicadas el reconocimiento de reacciones emocionales vía formas faciales son Eyeris: www.eyeris.ai y $A f$ fectiva: http://www.affectiva.com/ 
identificar los mejores medios para influenciarlos hacia una determinada decisión.

¿Cómo es posible que haya surgido este concepto tecnológico? ¿De dónde proviene la idea que a través de las manifestaciones físicas microfaciales podemos reconocer emociones y comportamientos o predecir pensamientos? Para responder a estas preguntas necesitamos conocer el fundamento teórico que sostiene este desarrollo tecnológico. Dicha teoría responde a un determinado punto de vista en el estudio de las emociones que se ha denominado "enfoque fisiologista de la emoción".

2. El SUSTRATO TEÓRICO DE LA MEDICIÓN DE LAS EMOCIONES VÍA FACIAL

En primer lugar, debemos destacar a Rosalind Picard (1997) como una de las investigadoras precursoras de la unión de la tecnología computacional con la dimensión emocional. Fundó, en esta línea, el Grupo de Investigación en Computación Afectiva en el Media Lab del Instituto Tecnológico de Massachusetts ${ }^{3}$, reuniendo a investigadores interesados en estudiar el uso de la informática para reconocer, entender e incluso para tener y expresar emociones.

La pregunta es ¿Cómo pudo emerger el interés de observar y medir emociones a través de las manifestaciones corporales mediante herramientas tecnológicas? Para responder a esta pregunta necesitamos retroceder a fines del s. XIX para encontrarnos con William James, pionero en el estudio de las emociones desde un enfoque fisiológico y científico. James a partir de sus investigaciones llegó a la convicción de que las emociones se explican como percepciones de determinados estados corporales y, por ende, las emociones están determinadas por las dimensiones biológicas que las originan y manifiestan:

Nuestra manera natural de pensar sobre esas emociones estándar es que la percepción mental de algún hecho provoca la disposición mental llamada emoción y que este estado mental da lugar a la expresión corporal. Mi tesis por el contrario, es que los cambios corporales siguen directamente a la percepción del hecho desencadenante y que nuestra sensación de esos cambios, según se van produciendo, es la emoción. El sentido común nos dice que nos arruinamos, estamos tristes y lloramos; que nos topamos con un oso, nos asustamos y corremos; que un rival nos ofende, nos enfadamos y golpeamos. La hipótesis defendida aquí afirma que este orden de la secuencia es incorrecto, que un estado mental no es inducido inmediatamente por el otro, que las manifestaciones

3 Disponible en: http://affect.media.mit.edu 
corporales deben interponerse previamente entre ambos y que una exposición más racional es que nos sentimos tristes porque lloramos, enfadados porque golpeamos, asustados porque temblamos [...] Si los estados corporales no siguieran a la percepción, esta última poseería una conformación totalmente cognitiva, pálida, incolora, carente de calor emocional. (James, 1884: 189-190)

Lo que está en juego en esta teoría es que las sensaciones de determinadas reacciones fisiológicas a estímulos constituyen la emoción y que, por tanto, el ejercicio cognitivo es posterior y secundario respecto a la relación directa que existe entre la reacción emocional originada en la percepción de los estados corporales que se producen en una situación reactiva. Es decir, aunque se vea un oso de improvisto ante nosotros y se decidiera huir, no es igual a sentir el estado emocional del miedo frente a ese acontecimiento. No huimos porque tenemos miedo, sino que tenemos miedo porque nuestro cuerpo huye. Según James, únicamente lo último es en propiedad una emoción. La evaluación del peligro del oso no genera por sí misma la emoción del miedo. Experimentamos miedo antes de la cognición, es decir, cuando nos percatamos de las alteraciones físico corporales que se han disparado frente a un oso. La emoción no es, por tanto, un ejercicio reflexivo sino una reacción y experimentación corporal básica, biológica e instintiva. Así mismo el fisiólogo Carl Lange (1912) ${ }^{4}$ continuará con esta misma línea de argumentación, insistiendo que lo específico de la emoción se encuentra en el componente muscular involuntario que la determina.

Así las cosas, tenemos que en la teoría de James-Lange la relación emoción y percepción se funda en un programa neuronal que coordina respuestas inmediatas, inconscientes y automáticas con los estímulos del entorno, por medio de mecanismos de señalización neuroquímicos tales como los sistemas endocrinos y nervioso autónomo. Concentrada en la dimensión fisiológica de la emoción, esta vertiente niega otras interpretaciones posibles acerca de las emociones que experimenta un sujeto, posición evidentemente marcada por las corrientes neopositivistas de fines del siglo XIX y comienzos del XX (Nussbaum, 2008: 61-62).

Por otra parte, Robert Zajonc (1980) afirma que ante la reacción que provoca una emoción existe un compromiso cognitivo mínimo. De la misma manera que un conejo reacciona y huye de la serpiente que la quiere atacar (Zanjonc, 1980: 156), los seres humanos poseen respuestas instintivas -sin cognición- de huida ante al peligro. Es decir, los seres humanos

\footnotetext{
4 Por esta cercanía teórica con James, es que se conoce a la teoría fisiologista como teoría "James-Lange".
} 
poseen los mismos mecanismos de percepción de sus emociones y están unidos por esa vía, directamente a los dispositivos de escape o ataque que determinan la vida en el reino animal. Es lo que expresa Leonard Berkowitz (1978) al insistir que las reacciones agresivas pueden ser simultáneas, o incluso, preceder a la experiencia consciente. La posición básica de Berkowitz es que una reacción agresiva emocional se ejecuta sin planes o pensamientos complicados, pues la determinación reactiva está fijada por la urgencia agresiva:

He sugerido que cuando las personas se enfrentan a un acontecimiento desagradable (bien algo inherentemente desagradable o algo que ellos interpretan negativamente), tienen una variedad de reacciones en sus pensamientos y recuerdos y a través de su cuerpo. Sugiero también que las respuestas ideacionales, fisiológicas y expresivo-motoras constituyen la base de la experiencia emocional. Los pensamientos y las ideas entran en escena presumiblemente después de que se han producido los sentimientos emocionales básicos. (Berkowitz, 1996: 107)

Dentro de esta teoría fisiologista de la emoción, nos encontramos con una referencia científica que sugiere poder medir emociones vía expresiones faciales. Es el caso de Silvan Tomkins (1988) y Paul Ekman (1984; 1992) que coinciden en su investigación en lo que se ha venido a denominar "programa afectivo". Dicho programa consiste en la idea que las emociones ponen al cuerpo en la condición de reaccionar y actuar en un espectro delimitado y claramente identificable mediante específicas expresiones faciales. Es decir, según estos autores, sin mediar cognición, la expresión corporal y facial está determinada de una manera innata y de acuerdo a esa determinación natural, sentimos como sentimos. En el programa de expresión facial de Silvan Tomkins se muestra, por ejemplo, que las emociones son respuestas faciales (Tomkins, 1988: 204-242). Es decir, para cada emoción se corresponde una figura facial. A partir de una correcta configuración muscular de una determinada emoción se puede dar lugar a los cambios fisiológicos que constituyen la experiencia subjetiva correspondiente a esa emoción. Mientras que Ekman por su parte, desarrolló en 1978 un Sistema de Codificación de la Actividad Facial (FACS), que le permitió medir y reconocer emociones a partir de movimientos faciales en términos anatómicos (Ekman, 2016: 32). Este sistema de Ekman fue posteriormente recogido y mejorado por científicos informáticos, logrando que la medición sea más automática y veloz (Ekman \& Rosenberg, 1997; Cohn, Zlochower, Lein \& Kanade, 1999). 


\section{EL COMPONENTE COGNITIVO DE LAS EMOCIONES}

A partir de las fuentes que hemos citado, podríamos pensar que la certeza de la medición de las emociones como efectos de reacciones innatas e involuntarias es la base de un negocio exitoso a todas luces por el grado de certeza científica con la cual cuenta el reconocimiento facial de las emociones. Es decir, aparentemente medir emociones vía facial y leerpredecir los comportamientos motivados emocionalmente es un procedimiento asertivo y científicamente respaldado. Sin embargo, este asunto no está libre de cuestionamiento pues existen otras posturas dentro de las mismas neurociencias y en otras áreas del conocimiento, que consideran que las emociones no se explican únicamente bajo su forma instintiva corporal y menos por mera manifestación facial. Existen las corrientes teóricas cognitivo-fisiológicas que comprenden las emociones desde su intencionalidad. Es decir, las emociones, desde este punto de vista, están vinculadas a la interpretación que el sujeto realiza sobre las manifestaciones emotivas que experimenta. Existen autores, científicos, psicólogos y filósofos que, sin negar el componente fisiológico de la emoción, no la reducen a aquella dimensión biológica. Estos autores prestan atención dentro de su enfoque a los procesos que permiten al sujeto convertirse en un ser pensante en pleno sentido. En otras palabras, bajo una perspectiva cognitiva, la emoción no puede ser explicada únicamente por su funcionamiento neuro-cerebral o por medio de sus expresiones físicas, faciales o corporales. Desde este punto de vista, no es totalmente correcta la afirmación de que las emociones sean irracionales (Damasio, 2010a; 2010b; 2015; LeDoux, 1999; Goleman, 2011; De Sousa, 1987; Goldie, 2000; Elster, 2002; Solomon, 1993).

Bajo este enfoque teórico diferente podemos justificar algunos puntos críticos respecto al negocio del reconocimiento facial de las emociones. Si estas empresas pretenden predecir comportamientos a partir de respuestas corporales sin considerar las dimensiones cognitivas asociadas a las emociones, obtenemos razones para dudar que estos emprendimientos estén vendiendo un producto cien por cien fiable. En el entramado afectivo, las emociones incorporan cogniciones que hacen de las manifestaciones emocionales actividades libres y por lo mismo, indescifrables e impredecibles. Para demostrarlo, se hace necesario sopesar el enfoque fisiologista a partir de teorías cognitivas y evaluativas de la emoción. Apelamos al criterio que indica que las emociones no se reducen a un aspecto meramente físico corporal, pues incluyen complejidades superiores.

Para obtener un lugar de habla apropiado, tenemos presente un principio rector que nos aporta Jon Elster para comprender mejor los procesos emocionales. Según Elster cuando hablamos de emociones estamos ante 
un proceso continuo (Elster, 2001: 14-15; Damasio, 2010b: 32) que va desde las emociones primarias a las emociones secundarias. Las emociones primarias son estados viscerales, respuestas automáticas ante estímulos, pues el compromiso cognitivo es mínimo y la intervención sociocultural prácticamente inexistente - como, por ejemplo, el temor ante un peligro, la ira o el asco. Mientras que las emociones secundarias son estados motivacionales, donde la reactividad corporal es escasa, el compromiso cognitivo es máximo y los aprendizajes culturales determinantes - como, por ejemplo, el amor o la compasión. La principal variable que marca el paso en este proceso desde la reactividad corporal automática hasta las emociones como estados motivacionales, la constituye el saber, sentir o percibir un estado, es decir, cuando se hacen cabalmente conscientes. Esta distinción de un proceso continuo nos ayuda a mantener la parte de veracidad de la perspectiva fisiologista, pero al mismo tiempo nos faculta para afirmar la necesidad de los componentes cognitivos, en tanto componentes preponderantes para la constitución de una emoción. Esta óptica nos permite afirmar una propuesta teórica que integra las dimensiones fisiológicas y cognitivas de la emoción. En virtud de ello, a nuestro juicio son tres los aspectos fundamentales dentro de un enfoque cognitivo fisiológico de las emociones.

\subsection{Emociones propiamente humanas: intencionalidad e interpretación}

Las consecuencias de las posturas fisiologistas reductivas nos conducen a consideraciones que parecen bastante extrañas. Por ejemplo, si una emoción se identifica exclusivamente a través de expresiones faciales determinadas una persona podría replicar las formas físicas que el rostro adquiere cuando está triste y con ello generar la tristeza. $\mathrm{O}$, si se quiere conocer la definición y la intensidad de la emoción del amor erótico, baste medir la presión arterial y sanguínea del órgano genital, tal como lo sugieren las investigaciones de James Weinrich (1980). Si se pueden medir las emociones vía micro expresiones faciales, ¿están agotadas todas las implicaciones dentro de la conducta de un individuo y, por tanto, se puede predecir cómo actuará? $\mathrm{O}$, por otro lado, ¿es posible admitir que la medición de una corriente sanguínea sea el modo de medir una emoción sexual o el amor de pareja? Según Martha Nussbaum tenemos aquí un problema de concepto, sin necesidad siquiera de considerar el aspecto metodológico, ni menos sus resultados que difícilmente pueden ser contundentes y cabalmente fehacientes (Nussbaum, 2008: 122). El concepto erróneo es que el afán científico experimental y fisiológico prescinde de abordar determinados problemas e hipótesis desde una perspectiva íntegral. Si se considera la más básica referencia a la propia humanidad, emerge la evidencia que 
las emociones son irreductibles a su materialidad física corporal en razón de la dimensión intencional que eidéticamente las constituye. Esto es, la emoción está inclaudicablemente referida a algo. La intencionalidad (Brentano, 1942; 2002) es un componente que da sentido completo a la emoción, pues incluye el modo como el sujeto interpreta las manifestaciones emocionales que experimenta, siempre en referencia a su yo propio (Ricoeur, 1986:20-25). Sin considerar dicha intencionalidad, es imposible leer los procesos fisiológicos que expresan emociones. La inclusión de la intencionalidad no se presenta como una negación absoluta de la perspectiva de base fisiológica inherente al proceso emocional sino como un llamado a que la explicación científica de la emoción incluya la intencionalidad dentro de sus análisis (Nussbaum, 2008: 123). No se trata entonces de negar el componente fisiológico propio de la emoción, se trata de ampliar el registro de análisis de esas expresiones para que se ajusten más a los fenómenos emotivos tal como se manifiestan.

La emoción puede expresarse de un modo físico o de otro, pero su definición pasará siempre por el aspecto cognitivo esencial que permite identificarla e interpretarla. En consecuencia, la diferencia que se puede establecer entre una emoción y otra no se hace cabalmente efectiva por medio de sus expresiones corporales, sino por el contenido cognitivo que la preside. Por ejemplo, una persona A puede expresar la alegría por medio de saltos continuos de júbilo, mientras que la persona $\mathrm{B}$ sonríe sin parar. Aunque se expresan manifestaciones corporales diferentes, se reconocerá que experimentan la misma emoción de alegría a través del contenido cognitivo que ambas personas expresan en la proposición: "Hemos aprobado el curso de Ética. Estamos muy alegres porque el esfuerzo que hicimos estudiando juntos, acabó teniendo sus frutos". Alguien escéptico podría objetar que hay diferencias de intensidad en la expresión física de las personas A y B, y que, por tanto, se podría medir y diferenciar la alegría en ambos casos. A lo que se puede responder: en todo nadie dudaría al identificar la emoción de alegría en ambas personas a partir de la preposición que han enunciado. $\mathrm{Y}$ si se requiriera identificar diferencias de intensidad bastaría con preguntar a las personas algo más sobre la emoción que experimentan, confirmando o no diferencias, pero ahora por una vía de interpretación del proceso cognitivo-lingüístico que define dicha emoción.

\subsection{Emociones unidas a razones}

A la visión que las emociones y los sentimientos constituyen un aspecto incontrolable del comportamiento y, por tanto, siempre opuesto a la razón, le ha seguido la perspectiva que las emociones y las razones constituyen un proceso unido y único. Se ha roto el "mito de las pasiones" 
según Robert C. Solomon (1993: 67-68. 142-152) o se ha terminado con el "error de Descartes", según Antonio Damasio (2015: 236. 332-337) ${ }^{5}$. Se ha logrado comprender que no hay ruptura ni dicotomía entre la razón y las emociones desde el punto de vista neurofísico, pero tampoco desde un aborde filosófico que se esfuerce por comprender la realidad de lo humano en la diversidad de sus manifestaciones.

Damasio nos muestra, por ejemplo, cómo cabe abordar el tema de las emociones en una perspectiva que integra la dimensión corporal y cognitiva, es decir, que, si bien las emociones tienen una base fisiológica, no significa que dejen de ser también fenómenos cognitivos:

Las emociones son programas complejos de acciones, en amplia medida automáticos, confeccionados por la evolución. Las acciones se complementan con un programa cognitivo que incluye ciertas ideas y modos de cognición, pero el mundo de las emociones es en amplia medida un mundo de acciones que se llevan a cabo en nuestros cuerpos, desde las expresiones faciales y las posturas, hasta los cambios en las vísceras y el medio interno.

Las sensaciones que sentimos de las emociones, por otro lado, son percepciones mixtas de lo que sucede en nuestro cuerpo y mente cuando manifiestan emociones. En lo relativo al cuerpo, las sensaciones sentidas son imágenes de acciones más que acciones, y el mundo de los sentimientos está hecho de percepciones consumadas en mapas cerebrales. Con todo, es preciso hacer una matización. Las percepciones que llamamos sentimientos de emociones constan de un componente especial que corresponde a los sentimientos primordiales de lo que hemos hablado antes. Esos sentimientos se basan en la relación única entre el cuerpo y el cerebro que la interocepción privilegia. [...] Los sentimientos de las emociones constituyen el siguiente paso y pisa los talones de la emoción, como el último y legítimo logro del proceso emocional, la

5 Damasio reprocha en Descartes errores a dos niveles. El primer nivel es su dualismo respecto a comprender los procesos mentales (res cogitans) separados totalmente del cuerpo (res extensa). Lo que tuvo un doble efecto negativo según Damasio, ya que por mucho tiempo se consideró poder estudiar la mente sin recurrir a la biología y a la neurobiología y, al contrario, se pensó que basta la neurobiología para explicar los procesos mentales. Para Damasio el cuerpo es a la mente como la mente es al cuerpo, es decir, un único proceso. La mente y el cuerpo humano requieren una perspectiva "organismica", esto es, concebir al organismo como un fenómeno holístico que posee procesos biológicos y mentales que interactúan permanentemente entre sí y con un ambiente externo físico y social. Por otra parte, Damasio alude a un error en Descartes de segundo nivel. Descartes y los racionalistas en general, habrían erigido una concepción de razón "elevada" como la más adecuada manera de tomar decisiones. Para los racionalistas la lógica cognitiva sería el único y mejor medio para conseguir los mejores resultados. La consecuencia adyacente es que las emociones tuvieron que quedar fuera pues el procesamiento racional debe desembarazarse de las pasiones si quiere ser asertivo. (Damasio, 2015:240) 
percepción mixta de todo lo que ha ocurrido durante la emoción: las acciones, las ideas, el estilo con que las ideas fluyen (lento o rápido, pegado a una imagen o cambiando rápidamente unas con otras). (Damasio, 2010a: 175-177)

La razón y las emociones trabajan colaborativamente para percibir la espesura significativa y significante que la realidad ofrece. A la realidad y sus dificultades se responde mediante ejercicios de razón y al mismo tiempo, a partir del "color" que las emociones permiten incorporar para calibrar con suficiencia los hechos. La idea central de Damasio es que, según sus estudios neurológicos y conductuales, las emociones y la razón entran en una relación de espiral. Así nació su conocida hipótesis del "marcador somático" (Damasio et al., 1996; 2015: 242) que sostiene la idea que las emociones ayudan en el proceso de razonamiento, en vez de perturbarlo. Damasio sostiene que los marcadores somáticos ayudan a la deliberación de un sujeto al permitirle resaltar opciones peligrosas o favorables y a eliminarlas o hacerse cargo de ellas rápidamente en la consideración consiguiente. Esta noción le permite a Damasio postular que el comportamiento personal y social requiere que los sujetos formen especies de "teorías" adecuadas a su propia mente y a la mente de los demás, para adaptar respuestas a las situaciones y los acontecimientos concretos. Si tenemos en cuenta este enfoque, se haría imposible intentar predecir cómo un sujeto actuará en la toma de una decisión teniendo en cuenta únicamente sus expresiones corporales y faciales.

\subsection{Emociones y conciencia de sí: el paso a los sentimientos}

Las respuestas emocionales automáticas, expresadas mediante expresiones corporales y faciales se pueden comprender como un vestigio evolutivo. Sin embargo, siguiendo el argumento evolucionista no es posible conceder que ese proceso se haya detenido y que las emociones se hayan fosilizado como meras respuestas físico-evolutivas. Las emociones, dentro del proceso evolutivo han llegado a constituirse en sentimientos:

El primer dispositivo, la emoción, permitió a los organismos responder de forma efectiva pero no creativamente a una serie de circunstancias favorables o amenazadoras en la vida: circunstancias "buenas para la vida" o "malas para la vida", resultados "buenos para la vida" o "malos para la vida". El segundo dispositivo, los sentimientos, introdujo una alerta mental para las circunstancias buenas o malas y prolongó el impacto de las emociones al afectar de manera permanente la atención y la memoria. Finalmente, en una fructífera combinación con los recuer- 
dos pasados, la imaginación y el razonamiento, los sentimientos condujeron a la aparición de la previsión y a la posibilidad de crear respuestas nuevas, no estereotipadas. (Damasio, 2010b: 81)

Apoya LeDoux esta perspectiva diciendo que los sentimientos incorporan a las respuestas emocionales aspectos cognitivos que nos permiten responder creativamente a nuestro entorno. Según LeDoux existe un elemento que permite entrar de lleno en el reconocimiento de los sentimientos a partir de una condición de suma relevancia: "La capacidad para tener sentimientos está directamente relacionada con la capacidad de tener conocimiento consciente del yo y de la relación de éste con el mundo" (1999: 138). En este sentido, los sentimientos son experiencias subjetivas por las que conocemos y percibimos nuestras emociones y al mismo tiempo, nos disponen a nuevas y más complejas relaciones con el medio.

Así comprendidos, los sentimientos se constituyen en la capacidad del sujeto de experimentar la realidad, de percibir cosas y hechos como nuestros $y$, en esta relación, los sentimientos nos permiten mentalizar las emociones que otorgan color a la realidad. Dicho en palabras de José Antonio Marina:

[...] los sentimientos son experiencias en las que el sujeto se encuentra implicado, complicado, interesado. El conocimiento mantiene una relación distanciada con las cosas: en eso consiste la objetividad. No enarbolo cuando veo un árbol. Me entristezco, en cambio, cuando veo un espectáculo triste. (2004: 77)

Los objetos forman una fusión con el yo, vía afectiva se comprometen con el yo. Es el origen de nuestro encuentro afectivo con la realidad. "El sujeto está en el sentimiento. Vive sentimentalmente, alumbra el mundo con su luz sentimental" (Marina, 2004: 77). En consecuencia, los sentimientos se constituyen en un determinado sello "personal" de nuestra vida afectiva pues se configuran primordialmente desde el sujeto que experimenta emociones. El sentimiento entonces se relaciona con aquello que expresamos cuando decimos "sentir" una emoción, es decir, cuando la emoción se convierte en una experiencia emocional mentalizada y consciente. La actividad cognitiva en este sentido se hace preponderante para quien experimenta emociones, convirtiéndolas en sentimientos. 
4. CONSIDERACIONES ÉTICAS DEL NEGOCIO DE RECONOCIMIENTO FACIAL DE LAS EMOCIONES

Después de estas distinciones y críticas al modelo teórico en que se basan las startups que venden predicciones de comportamiento mediante mediciones emocionales por vía expresiones físicas y faciales, pensamos estar en condiciones de elaborar y proponer ahora una valoración ética.

El neuromarketing (también denominada neuropublicidad) ofrece debates éticos muy interesantes (Feenstra, 2013; Belden, 2008). Se han expresado visiones críticas respecto a la posible pérdida de autonomía del consumidor mediante procesos de invasión de la publicidad en la intimidad de las personas e incluso de la manipulación de la mente para fines de lucro. También emergen problemas éticos cuando a través del neuromarketing se puede aumentar las ventas de productos que atentan contra la salud pública, como es el caso del tabaco, la comida chatarra o el alcohol. Es perfectamente posible que las nuevas estrategias publicitarias basadas en los conocimientos de la neurociencia ofrezcan medios ilegítimos para usos inadecuados en la medida que pueden entorpecer el libre ejercicio de la voluntad y la libertad, oponiéndose con ello a criterios de justicia (Wilson, Gaines \& Hill, 2008).

Las mediciones emocionales mediante reconocimiento facial agregan sus propios problemas éticos que resultan evidentes incluso para su principal exponente. El mismo Paul Ekman, creador del FACS (Facial Action Coding System), método que permite leer las emociones mediante movimientos musculares del rostro, manifiesta su preocupación por haber creado un "monstruo" (Dwoskin \& Rusli, 2015). Está consiente que las empresas que han utilizado su investigación como columna vertebral están amasando, mediante softwares especializados, una enorme base de datos visual de emociones humanas, buscando patrones que pueden predecir reacciones emocionales y comportamientos a una escala masiva. Le preocupa a Ekman que esa información sea utilizada responsablemente sin infringir la privacidad personal. Pues ya existen antecedentes de utilización de estos softwares en cámaras de seguridad dirigidas a la cara de las personas que entran en establecimientos comerciales para identificar sus emociones de entrada o se usan en EEUU aplicándolos en interrogatorios policiales y de seguridad nacional. Se sabe que mediante estos procedimientos se puede incluso monitorear secretamente a los trabajadores o a los candidatos a un puesto laboral, entre otras aplicaciones. El peligro es que esta tecnología puede revelar las emociones sin el consentimiento de las personas y además los datos obtenidos puede ser mal interpretados perjudicando a los implicados. Sin una regulación efectiva y transparente de estos procedimientos mediante una legislación actualizada que establezca 
límites de uso de la información de los afectados, difícilmente estas tecnologías de reconocimiento facial podrían obtener legitimidad y confianza social. Para llegar, a esa confianza evidentemente hay que favorecer reflexiones éticas que tengan en cuenta no solo las posibles consecuencias positivas en la implementación de esta tecnología (como en el caso del control de la pandemia), sino también los riesgos que incuban.

Por nuestra parte, queremos ofrecer tres consideraciones éticas para reflexionar sobre este asunto, y que derivamos de los tres aspectos que nos aportan las teorías cognitivas de la emoción y que hemos sintetizado en el punto tres de este artículo.

\subsection{Una visión integral de la complejidad del ser humano}

A nuestro modo de ver el principal problema ético al que se enfrentan las empresas que se dedican al reconocimiento facial de las emociones es la reducción que realizan del comportamiento humano en general y del consumidor en particular a comprensiones unidimensionales. En otras palabras, cuando estas empresas consideran al sujeto como un objeto que toma decisiones basados exclusivamente en emociones, entendidas como automáticas e irreflexivas, no prestan atención -ni desean hacerlo- a la dimensión cognitiva presente en todo proceso de decisión y que erige a cualquier elección en plenamente libre y voluntaria. Es bajo esta visión reduccionista, cuando el fantasma de la manipulación de las personas se vuelve una tentación concreta cuando el fin es aumentar los beneficios económicos.

Por otra parte, estas perspectivas reduccionistas se basan en la convicción de conseguir determinados indicadores musculo-faciales fidedignos que validen a tal punto una emoción que se conviertan en su exclusivo elemento definitorio. Se pretende, mediante el procedimiento del reconocimiento facial que el ejercicio muscular detectado corresponda directa y fehacientemente con la emoción que expresa para predecir un comportamiento específico. Si bien es posible que esta concordancia se de en emociones primarias o básicas — como la alegría, la ira o el asco, por ejemplo(LeDoux, 1999: 124; Damasio, 2015: 189-193), no se ve que dicha concordancia sea fehaciente en emociones secundarias o complejas - como la ansiedad o la frustración (LeDoux, 1999: 124-125; Damasio, 2015: 195). Las emociones poseen un programa cognitivo que incluye intencionalidad, ideas, creencias, aprendizajes sociales y determinaciones culturales. Esto es, incluyen pensamientos en un sentido amplio que configuran representaciones tan complejas que no se puede esperar se vean reflejadas y agotadas en expresiones faciales determinadas. Las emociones, en este sentido, 
poseen un carácter disposicional aprendido y no tanto innato. Las emociones también se generan a partir de representaciones de experiencia subjetivas y conllevan respuestas de acción adecuadas a dichas representaciones (Damasio, 2015: 158). Evidentemente esta complejidad reduce a su mínima expresión la posibilidad de una interpretación certera cuando se intenta leer microexpresiones.

Es una responsabilidad ética de estas empresas ofrecer un producto que responda a un marco interpretativo más sistemático que asegure a sus clientes que se les está vendiendo un producto con un valor de cambio justo. Considerando lo que aquí hemos dicho, más bien parece ser una falacia pretender vender inferencias de estados mentales y predicciones de comportamientos con la sola observación y medición de movimientos musculares del rostro.

\subsection{Libertad como autonomía}

La comprensión autómata de las emociones para las decisiones de compra en la que se basan las startups posee todavía un sustrato más profundo. Como hemos mencionado, los avances de las neurociencias en el área de la economía han descubierto que las decisiones de consumo están las más de las veces determinadas por el factor emocional. Esta conclusión sólo es posible si se aplica un modo específico de comprender la libertad. Cuando se dice que las emociones son preponderantes para la elección, subyace una comprensión de libertad como una mera satisfacción de deseos. Sin embargo, la libertad no es solamente el medio que permite satisfacer determinados deseos o necesidades de consumo, sino que la libertad es autonomía, en tanto proceso de auto legislación que la razón permite (Kant). La libertad es la capacidad que los ciudadanos tienen para erigirse en dueños sensatos de la construcción de su propia identidad moral. Esa identidad se modula por la satisfacción de deseos, pero también, como expresa Adela Cortina, por la búsqueda de encarnar un ideal normativo que se razona, movilizando sentimientos y actitudes:

Ciudadanía obliga, y obliga en esa dimensión humana que es la de ser consumidor a elegir la forma de consumo atendiendo a criterios, y no a otros, favoreciendo actitudes y debilitando otras, proponiendo estilos de vida en la línea de lo que debería ser. Una "teoría normativa" del consumo se hace necesaria, una teoría acorde con esa noción de ciudadanía densa, de la que ya no podemos apearnos (Cortina, 2002: 38) 
Elevar la libertad a una autonomía significa entre otras cosas, hacerse responsable. Las emociones en este sentido no son irracionales. Las emociones permiten "saborear" motivaciones, creencias, aprendizajes, valores, opciones de vida buena. Las elecciones de consumo que se guían por este pleno ejercicio de libertad y autonomía moral nos hacen auténticos seres racionales y no marionetas de un proceso económico, productivo y publicitario.

\subsection{La relevancia de consumidores conscientes}

Las emociones dentro un enfoque fisiologista-cognitivo, contribuyen a una evaluación del presente que viene desde el pasado y nos moviliza para el futuro. Dada su capacidad evaluadora las emociones no son desnudas de sentido. Nos proporcionan el dinamismo que vitalizan nuestras acciones. Nos abren al mundo de los valores que se manifiestan como un proyecto por realizar. Sólo bajo esta conexión las emociones disponen la experiencia de un razonamiento en toda su amplitud práctica. Las emociones que son adecuadas, configuran y fortalecen razonamientos prácticos adecuados. Así un gusto equivocado se corrige mediante argumentos y reflexiones ulteriores. En virtud de ello, las emociones nos permiten contar con un piso afectivo que puede elevarse a acto intelectivo práctico. Dado que el placer de lo agradable no es suficiente para las decisiones correctas, aspiramos a una estatura moral mayor que otorgue sentido a nuestras acciones, no guiados por la pura satisfacción en la epidermis de los sentidos. Es la posibilidad que otorga plena conciencia de lo que somos y de lo que nuestras emociones expresan cuando entran en juego en nuestras elecciones.

\section{CONCLUSIONES}

En el actual contexto de pandemia, se convierte en un imperativo ético la consideración crítica de la implementación del reconocimiento facial como medida de control digital sobre los ciudadanos. Si bien, anima a los estados que lo implementan la finalidad de la salud pública, existen varios aspectos éticos importantes que se requieren sopesar para guiar la puesta en marcha de estos procedimientos tecnológicos. Entre otros nudos problemáticos encontramos: el uso de datos biométricos privados, la ausencia de consentimiento de los afectados, el sobre control de las libertades individuales, como también los riesgos de discriminación por errores en el reconocimiento o abusos de poder sobre las personas.

Por otro lado, los estados requieren ser responsables con los recursos públicos en la adquisición de estos servicios de reconocimiento facial, pues 
su efectividad todavía no está suficientemente respaldada. Recientemente se han informado en diferentes medios de comunicación problemas en las mediciones biométricas y errores con los algoritmos a causa del uso de las mascarillas. Estos errores se explican porque se ha reducido a la mitad la capacidad de identificación de los rostros. Esta situación ha motivado a las empresas proveedoras a desarrollar nuevos algoritmos que incluyan las mascarillas, reduciendo el área facial de identificación. Obviamente con este problema provocado por la contingencia pandémica, se reduce aún más la efectividad y la precisión de la información recabada mediante esta tecnología.

En este sentido, un emprendimiento comercial responsable exige que el servicio que se ofrece asegure que el cliente obtiene efectivamente lo que compra. A partir de nuestras consideraciones hemos sopesado que es posible dudar sobre la predicción de comportamientos, basándose en la observación y medición de emociones sobre manifestaciones faciales y corporales. Nos apoyamos principalmente en la idea que las emociones dentro del complejo evolutivo, no se corresponden unívocamente a puras reacciones instintivas automáticas. Las emociones han llegado a ser componentes fundamentales en la decisión libre, en la medida que las emociones interactúan permanentemente con razonamientos, ideas y valores. Esta compleja vinculación de emociones y razonamientos se constituye en el sustrato que permite que los seres humanos sean sujetos libres, voluntarios y plenamente responsables.

Por otro lado, hemos tratado de mostrar que esta misma complejidad dificulta la interpretación de comportamientos en base a meras reacciones físicas, dado que cualquier toma de decisión incluye procesos cognitivos ulteriores presentes en toda decisión y acción. De modo que no se puede suponer asertividad de decisiones que se puedan predecir a partir de reacciones emotivas observadas en un reconocimiento facial. En conclusión, dudamos que las empresas dedicadas al reconocimiento facial de las emociones estén ofreciendo un producto garantizado. Y todavía más, si consideramos las perspectivas teóricas que incluyen los procesos cognitivos en la explicación de las emociones, tendemos a pensar que estas empresas están comercializando su servicio mediante una publicidad engañosa. Las teorías cognitivistas de la emoción permiten postular precisamente la imposible capacidad de leer conductas a través de las facciones emocionales pues la dimensión cognitiva, parte sustantiva de la emoción hecha sentimiento, complejiza la predicción de un comportamiento humano.

Por último, resaltamos la idea que la libertad no se agota en un ejercicio de satisfacción de deseos comerciales. Un ejercicio pleno de libertad está encaminado a la responsabilidad y la autonomía de los agentes. Prever 
y hacer negocios pensando en que los seres humanos pueden ser conducidos merced a su supuesta irracionalidad emotiva, siguiendo únicamente intereses económicos y/o políticos se constituye al final en una minusvaloración, una flagrante y muy poca ética manipulación.

\section{REFERENCIAS}

Belden, S.R.A. (2008). Neuroeconomics and neuromarketing. Practical applications and ethical concerns. Journal of Mind Theory, (2), 249-255.

Berkowitz, L. (1978). Do we have to believe we are angry with someone in order to display "angry" aggresion toward that person? En L. Berkowitz (comp.), Cognitive Theories in Social Psychology: Papers from Advances in experimental Social Psychology (pp. 455-463). New York: Academic Press.

Berkowitz, L. (1996). Agresión. Causas, consecuencias y control. Bilbao: Desclée de Brouwer.

Brentano, F. (1942). Psicología. Buenos Aires: Schpire.

Brentano, F. (2002). El origen del conocimiento moral. Madrid: Tecnos.

Cisneros, A. (2015). Neuromarketing y neuroeconomía. Código emocional del consumidor. Hispamérica Books.

Cohn, J. F., Zlochower, A. J., Lien, J., \& Kanade, T. (1999). Automated face analysis by feature point tracking has high concurrent validity with manual FACS coding. Psychophysiology, 36(1), 35-43. https://doi.org/10.1017/s0048577299971184

Conill, J. (2012). Neuroeconomía y neuromarketing ¿Más allá de la racionalidad maximadora? En A. Cortina (Ed.), Guía Comares de Neurofilosofía Práctica (pp.39-64). Granada: Comares.

Cortina, A. (2002). Por una ética del consumo. La ciudadanía del consumidor en un mundo global. Madrid: Taurus.

Cortina, A. (2011). Neuroética y neuropolítica. Sugerencias para la educación moral. Madrid: Tecnos.

Damasio, A. R., Everitt, B. J., Bishop, D., Roberts, A. C., Robbins, T. W., \& Weiskrantz, L. (1996). The somatic marker hypothesis and the possible functions of the prefrontal cortex. Philosophical Transactions of the Royal Society B: Biological Sciences, 351(1346), 1413-1420. http://doi.org/10.1098/rstb.1996.0125

Damasio, A. (2010a). Y el cerebro creó al hombre. ¿Cómo pudo el cerebro generar emociones, sentimientos, ideas y el yo? Barcelona: Destino.

Damasio, A. (2010b). En busca de Spinoza. Neurobiología de la emoción y los sentimientos. Barcelona: Crítica.

Damasio, A. (2015). El error de Descartes. La emoción, la razón y el cerebro humano. Barcelona: Destino.

De Sousa, R. (1987). The Rationality of Emotion. Cambridge: The MIT Press.

Dimigen, O., Sommer, W., Hohlfeld, A., Jacobs, A. M., \& Kliegl, R. (2011). Coregistration of eye movements and EEG in natural reading: analyses and 
review. Journal of Experimental Psychology. General, 140(4), 552-572. https://doi.org/10.1037/a0023885

Dwoskin, E., \& Rusli, E. (2015). La tecnología que lee las emociones ocultas en su rostro. The Wall Street Journal (2 de febrero). Disponible en: https://www.wsj.com/articles/la-tecnologia-que-lee-las-emociones-ocultas-en-su-rostro-1422920315

Ekman, P. (1984). Expression and the nature of emotions. En K.R. Scherer \& P. Ekman (Eds.), Approaches to emotions (pp.319-344). Hillsdale, New Jersey: Erlbaum.

Ekman, P. (1992). Cómo detectar mentiras: una guía para utilizar en el trabajo, la politica y la pareja. Barcelona: Paidós.

Ekman, P. (2015). El rostro de las emociones. Cómo leer las expresiones faciales para mejorar sus relaciones. Barcelona: RBA.

Ekman, P. \& Rosenberg, E.L. (1997). What the Face Reveals: Basic and Aplied Studies of Spontaneous Expression Using the Facial Action Coding System (FACS). Oxford: University Press.

Elster, J. (2001). Sobre las pasiones. Emoción, adicción y conducta bumana. Barcelona: Paidós.

Elster, J. (2002). Alquimias de la mente. La racionalidady las emociones. Madrid: Paidós.

Feenstra, R. (2013). La irrupción de la neuropublicidad y sus debates éticos. Daimon Revista Internacional de Filosofia, (59), 45-56.

García J. L. (2016). Una startup de neuropolítica busca $400.000 €$ para lanzarse al mercado inglés. Levante. El Mercantil Valenciano (17 de marzo). Disponible en: https://www.levante-emv.com/economia/2016/03/17/startup-neuropolitica-busca-400000--lanzarse-mercado-ingles/1392878.html

Gigerenzer, G. (2008). Decisiones instintivas. La inteligencia del inconsciente. Barcelona: Ariel.

Goldie, P. (2000). The emotions. A Philosophical Exploration. Oxford: Clarendon Press.

Goleman, D. (2011). Inteligencia emocional. Barcelona: Kairós.

James, W. (1884). What is a Emotion? Mind, 9(34), 188-205.

Klarick, J. (2016). Véndele a la mente, no a la gente. México: Paidós Empresa

Lange, C.G. (1912). The Mechanism of the emotions. En E. Rand (Ed.), The Classical Psychologists (pp. 672-684). Boston: Houghton Mifflin.

Lee, N., Broderick, A. J., \& Chamberlain, L. (2007). What is "neuromarketing"? A discussion and agenda for future research. International Journal of Psychophysiology: official journal of the International Organization of Psychophysiology, 63(2), 199204. https://doi.org/10.1016/j.ijpsycho.2006.03.007

LeDoux, J. (1999). El cerebro emocional. Barcelona: Ariel/Planeta.

Lindstrom, M. (2010). Buyology: verdades y mentiras sobre por qué compramos. Ediciones Gestión 2000.

Marina, J. A. (2004). El laberinto emocional. Barcelona: Anagrama.

MacInnes, C. \& Steidl, P. (2016). Shopper Marketing: Neuromarketing Strategies to win the battle at the shelf. Createspace Independent Publishing Platform. 
Nussbaum, M. (2008). Paisajes del Pensamiento: la inteligencia de las emociones. Barcelona: Paidós.

Picard, R. (1997). Afective Computing, MA, USA: MIT Press Cambridge.

Racine, E., Bar-Ilan, O., \& Illes, J. (2005). fMRI in the public eye. Nature reviews. Neuroscience, 6(2), 159-164. https://doi.org/10.1038/nrn1609

Ricoeur, P. (1986). Lo voluntario y lo involuntario I: el proyecto y la motivación. Buenos Aires: Editorial Docencia.

Solomon, R. (1993). The passions: Emotions and the Meaning of Life. Hackett Indianapolis: Publishing Company.

Tomkins, S.S. (1988). Affect. Imagery. Consciousness (Vol. 1). New York: Springer Publishing Company.

Weinrich, J.D. (1980). Toward a Sociobiological Theory of the Emotions. En Plutchik y Kellerman (Eds.), Emotion: Theory, research, and experience. (Vol. 1, pp. 113-138). New York: Academic Press.

Wilson, J., Gaines, J. \& Hill, R. (2008). Neuromarketing and consumer free will. The Journal of Consumer Affairs, 42(3), 389-410. https:/ /doi.org/10.1111/j.1745-6606.2008.00114.x

Zajonc, R. B. (1980). Feeling and thinking: Preferences need no inferences. American Psychologist, 35(2), 151-175. https://doi.org/10.1037/0003066X.35.2.151 